\title{
Health Informatics in Developing Countries: A Review of Unintended Consequences of IT Implementations, as They Affect Patient Safety and Recommendations on How to Address Them
}

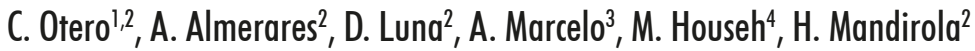 \\ 1 IMIA Health Informatics for Development Working Group Chair \\ 2 Health Information Department. Hospital Italiano de Buenos Aires, Argentina \\ 3 IMIA Health Informatics for Development Working Group Co-Chair, National Telehealth Center, \\ University of the Philippines Manila, Philippines \\ ${ }^{4}$ College of Public Health and Health Informatics, King Saud Bin Abdulaziz University for Health \\ Sciences, Riyadh, Saudi Arabia
}

\begin{abstract}
Summary
Background: Patient safety concerns every healthcare organization. Adoption of Health information technology (HIT) appears to have the potential to address this issue, however unanticipated and undesirable consequences from implementing HIT could lead to new and more complex hazards. This could be particularly problematic in developing countries, where regulations, policies and implementations are few, less standandarized and in some cases almost non-existing. Methods: Based on the available information and our own experience, we conducted a review of unintended consequences of HIT implementations, as they affect patient safety in developing countries.

Results: We found that user dependency on the system, alert fatigue, less communications among healthcare actors and workarounds topics should be prioritize. Institution should consider existing knowledge, learn from other experiences and model their implementations to avoid known consequences. We also recommend that they monitor and communicate their own efforts to expand knowledge in the region.
\end{abstract}

\section{Keywords}

Unintended consequences, patient safety, health information system, information technology, developing countries

Yearb Med Inform 2016:70-2

http://dx.doi.org/10.15265/IY-2016-028

Published online November 10, 2016

\section{Introduction}

Over the past years we have seen an increase in health information technology implementations (HIT) [1] with the Institute of Medicine in the United States stating that HIT is an essential tool to improve healthcare costs, patient safety and the quality and equity of care [2-4]. However in many cases, implementations have been hampered by unintended consequences that can not only limit the value of the implementation but also negatively affect the quality of care and patient safety.

The potential to generate new problems by implementing HIT is not a minor detail, there are numerous reports indicating how HIT, especially from failed implementations, can become a new source of risk for the patient. The errors induced by technology such as those generated by poor designs, changes in workflow and lack of training are so important and frequent that they have been defined as "technological iatrogenic "or "e-iatrogenesis" [5-6].

Developing nations suffer from many limitations when it comes to implementing HIT such as the lack of adequate infrastructure or funding; no nationwide e-Health agendas to achieve sustainable implementations; public uncertainty with respect to privacy and security; the difficulties in achieving interoperability and the absence of a trained workforce in health informatics [7]. These limitations have led to many organizations having to purchase and in some cases relay on precarious systems. As an example, in some Arab countries, outside the Gulf Cooperation Council (GCC), open source medical records or local companies have led the initiative in supporting healthcare organizations develop and implement Electronic Medical Record (EMR). Although, there have been benefits in terms of cost savings and creating local employment, The lack of technical know-how or development maturity have caused failure in implementation. Simple issues, such as not using the fourth name of the patient (i.e., first name, father name, grandfather, name and family name), were neglected at times causing misidentification of patients. Issues as no customer support and no consideration for Electronic Medical Record usability, at times, caused system failure [8]. This has created new unintended security concerns that impact patient safety and quality of care.

A review of the unintended consequences of IT implementations, as they affect patient safety is needed. 


\section{Methodology}

Taking into account the described reality, we conducted a non-systematic review of the literature. Based on the available information and our own experience we determined four broad categories related to unintended consequences of IT implementations that compromise patient safety:

1) User dependency on the system

2) Alert fatigue

3) Changes in Communication Patterns

4) Workarounds

This review article describes unintended consequences that compromise patient safety and that might be faced, particularly, by developing countries with possible ways to address them, in the hope of assisting in preventing their occurrence.

\section{Results}

\section{User Dependency on the System}

This unintended outcome refers to the risk of having users increasingly dependent on systems, abandoning their own criteria and committing errors. A perfect example of this is the case presented by MacDonald et al [9], were healthcare personnel became dependent of patient bar-coding identification before doing a procedure, and so when an error was committed when placing a wristband, and no oral or other form of confirmation of patient identity was sought, the way was paved for errors. This example is a good representation of the danger of thinking a system with little human intervention. As HIT implementation grows in developing nations (or elsewhere) we must not abandon our own existing criteria and practice. The redundancy of seeking other forms of confirmation before taking action, however time consuming, might prevent harm. We would be doing ourselves and our patients a disfavor if we were to fall into the preconception that the "computers" can do no wrong.

Overreliance on technological solutions, without parallel efforts to institute and enforce appropriate processes of care may only provide the illusion of safety.
We believe that to mitigate the dependency effect, institutions should devote themselves to developing workflows with appropriate redundancies, communicating systems limitations to users and monitoring process.

\section{Alert Fatigue}

The use of Alerts to support clinicians in their decision making is arguably one of the most discussed issues related to HIT, with evidence that supports an improvement in selected clinical surrogate outcomes and changes in process outcomes when using these systems [10-11]. Regrettably the potential of alerts has been hampered by alert fatigue. The term alert fatigue describes how workers become desensitized to safety alerts, and as a result ignore or fail to respond appropriately to such warnings [12]. The alert fatigue phenomenon has been a major unexpected consequence of decision support systems; it occurs for various reasons, firstly the sheer number of alerts, and the perceived fact that the vast majority of them are inconsequential. Furthermore, the way these alerts are design, in the sense of how much they interfere with a process and how well curated and accurate the knowledge base that supports them is, play a major role in the way healthcare workers respond to alerts [12-13].

The possibility of errors that derive from alert fatigue is not to be taken lightly. Inexperience with decision support systems in developing nations could be a factor that augments the possibility of errors associated with alerts. Also the lack of standards that affect quality of care, particularly in developing nations, could be an issue when creating the knowledge base that supports the alerts [14]

There are several ways to address this unexpected consequence. Firstly, much care should be placed in the design of the alert, for instance fatigue would be reduced by making non-interruptive all non-critical or low-severity alerts [15]. Furthermore the amount of alerts that are displayed should be kept at a minimum. Every requirement for alert should be carefully considered and the content of it should be curated and discussed with those who are going to be affected by it, stakeholders must participate early as possible in the development of the alert and be explicit about its value.

\section{Changes in Communication}

HIT has been fundamental in improving communication between providers and also patients. This improvement was achieve, for instance, by replacing illegible prescriptions with computerized provider order entry (CPOE) and unifying different sources of information into one unique electronic chart that can be available to all. However this wasn't without unintended consequences, new patterns in communication ended up also undermining vital communication flows among clinicians and with patients [16]. We have seen how the introduction of IT has led to a decline of vital interaction among care providers. We no longer have to walk to the nursing station to prescribe medications; we simply state it in the CPOE and expect the order to be followed. Less interaction is follow by the elimination of redundant checks or informal verifications that came with that needed exchange, we have lost an opportunity to catch errors and prevent harm [17].

Efforts should be made to maintain and improve communication in healthcare. Quality care demands close collaboration between healthcare workers and patients. New opportunities to add patients as auditors of their own health could be valuable when trying to prevent medication errors. Opening the medical records in real time could help catch errors and also improve relationship between patients and physicians [18]. New communication channels among providers are needed but more importantly we need to encourage and support clinicians to sustain their current interactions. To this end, nations planning to increase their implementation must put close attention in understanding current workflows and how they will be affected.

\section{Workarounds}

The line goes "nature will find a way..." well busy unhappy providers will also find a way to surpass what bothers them. For every new process that is deemed complicated or counterintuitive a workaround exits. 
With user that suffers from the cognitive overload caused by systems that overemphasize structured and "Complete" Information Entry or Retrieval and no consideration of user center principles as they affect human-computer interaction [19] the existence of workarounds, although unintended, is understandable. Even if HIT is not the sole responsible of this alternative flux of work, it still plays a big part, that is way most of the publications that have looked into the phenomenon has been done in the past few years [18].

We have seen paper persisting in the form of notes or clips and have seen them in clinicians or nurses pockets and also around monitors or in the floor [17]. This notes, that for the most part, are meant to assist in some cognitive process, maintain all the problems paper based charts had, but what is worst they do so without the formal validity of paper records. Is not only documentation that creates workarounds, cumbersome process set in motion by the introduction of HIT, such as patient identification requirements and test ordering contribute to clinicians and nurses creating this temporary "fixes". Workarounds related to patient identification and drug administration have been found to be significantly associated with making errors that end up compromising patient safety [20]

There are many ways to prevent workaround to develop. Hospital managers or those in charge of implementing HIT should pay careful attention into existing workflows, understanding, documenting and testing the impact of changing them[21]. Process should be reviewed and monitor, as the existence of workarounds could go unnoticed. It would also be wise to pay close attention to usability when designing interfaces, as user satisfaction together with a thorough understanding of user workflow will avoid the need for the workaround itself.

\section{Conclusion}

What happens by chance is not preventable and will always elude our finest methods of prevention. Unintended consequences of the past are no chance; they can be studied, analyzed and prevented. In this paper we have look at different types of these unintended consequences as they can impact patient safety. User dependency on the system, Alert fatigue, less communications among healthcare actors and workarounds are all symbols of the failure of HIT to achieve the intended full potential.

For all the difficulties and disadvantages we suffer from in developing nations, there is still one very significant advantage, learning from others. Hospital managers, policy makers and implementers should look into this review as a first step in understanding the occurrence of unintended consequences. Institution should model their implementation to avoid known consequences to happen and also monitor and communicate on their own implementation as well as lessons learned.

\section{References}

1. World Health Organization Global Observatory for eHealth. Building foundations for eHealth : progress of member states : report of the WHO Global Observatory for eHealth. Geneva, Switzerland: World Health Organization; 2006.

2. Dick RS, Steen EB, Detmer DE; Committee on Improving the Patient Record, Institute of Medicine. The computer based patient record: an essential technology for health care. Washington (DC): National Academy Press; 1997.

3. Kohn LT, Corrigan JM, Donaldson MS. To err is human: building a safer health system. Washington (DC): National Academy Press; 2000.

4. Institute of Medicine, Committee on Quality of Health Care in America. Crossing the quality chasm: a new health system for the 21 st century. Washington (DC): National Academy Press; 2001.

5. Palmieri PA, Peterson LT, Corazzo LB. Technological iatrogenesis: the manifestation of inadequate organizational planning and the integration of health information technology. Adv Health Care Manag 2011;10:287-312.

6. Weiner JP, Kfuri T, Chan K, Fowles JB. "e-Iatrogenesis": the most critical unintended consequence of CPOE and other HIT. J Am Med Inform Assoc 2007;14:387-8; discussion 389.

7. Luna D, Almerares A, Mayan JC, González Bernaldo de Quirós F, Otero C. Health Informatics in Developing Countries: Going beyond Pilot Practices to Sustainable Implementations: A Review of the Current Challenges. Healthc Inform Res 2014;20(1):3-10.

8. Alsadan M, El Metwally A, Anna ALI, Jamal A, Khalifa M, Househ M. Health Information Technology (HIT) in Arab Countries: A Systematic
Review Study on HIT Progress. J Health Inform Dev Ctries 2015;9(2).

9. McDonald CJ. Computerization Can Create Safety Hazards: A Bar-Coding Near Miss. Ann Intern Med 2006;144(7):510-6.

10. Borbolla D, Taliercio V, Schachner B, Luna D, Gonzalez Bernaldo de Quiros F. Isolation of patients with vancomycin resistant enterococci (VRE): efficacy of an electronic alert system. Stud Health Technol Inform 2012;180:698-702.

11. Bayoumi I, Al Balas M, Handler SM, Dolovich L, Hutchison B, Holbrook A. The effectiveness of computerized drug-lab alerts: a systematic review and meta-analysis. Int J Med Inform 2014;83(6):406-15.

12. Agency for healthcare research and quality. Alert Fatigue. https://psnet.ahrq.gov/primers/primer/28/ alert-fatigue (accessed January 4 2016).

13. Lee EK, Mejia AF, Senior T, Jose J. Improving Patient Safety through Medical Alert Management: An Automated Decision Tool to Reduce Alert Fatigue. AMIA Ann Symp Proc 2010;2010:417-21.

14. Thaver I H, Harpham T, McPake B, Garner T. Private Practitioners in the Slums of Karachi: What Quality of Care Do They Offer? Soc Sci Med 1998;46(11):1441-49.

15. Shah NR, Seger AC, Seger DL, Fiskio JM, Kuperman GJ, Blumenfeld B. Improving acceptance of computerized prescribing alerts in ambulatory care. J Am Med Inform Assoc 2006 Jan-Feb; 13(1):5-11.

16. Ash JS, Berg M, Coiera E. Some Unintended Consequences of Information Technology in Health Care: The Nature of Patient Care Information System-related Errors. J Am Med Inform Assoc 2004;11(2):104-12.

17. Campbell E, Sittig D, Ash J, Guappone K, Dykstra $R$. Types of unintended consequences related to computerized provider order entry J Am Med Inform Assoc 2006;13(5):547-56.

18. Walker J, Meltsner M, Delbanco T. US experience with doctors and patients sharing clinical notes. BMJ 2015 Feb 10;350:g7785. Available from: http://www.ncbi.nlm.nih.gov/pubmed/25670182

19. Ash JS, Berg M, Coiera E. Some Unintended Consequences of Information Technology in Health Care: The Nature of Patient Care Information System-related Errors. J Am Med Inform Assoc 2004;11(2):104-12.

20. Westbrook JI, Rob MI, Woods A, Parry D. Errors in the administration of intravenous medications in hospital and the role of correct procedures and nurse experience. BMJ Qual Saf 2011;20(12):1027-34.

21. Meeks DW, Smith MW, Taylor L, Sittig DF, Scott JM, Singh H. An analysis of electronic health record-related patient safety concerns. J Am Med Inform Assoc 2014;21(6):1053-9.

\section{Correspondence to:}

Dr. Carlos Martín Otero

Departamento de Informática en Salud

Hospital Italiano de Buenos Aires

E-mail: carlos.otero@hospitalitaliano.org.ar 